\title{
Predictive Current Control vs. PI Control for Surface Mounted Permanent Magnet Machines
}

\author{
Lynn Verkroost, Student Member, IEEE, Joachim Druant, Hendrik Vansompel, \\ Frederik De Belie, Member, IEEE and Peter Sergeant, Member, IEEE
}

\begin{abstract}
This article presents a comparative study of a standard PI controller with three more advanced predictive current control schemes, applied to a surface mounted permanent magnet synchronous machine fed by a two-level voltage source inverter. The three predictive current controllers are: finite-set model based predictive control, deadbeat control, and a combination of both previous algorithms. Key performance indicators concerning control- and voltage quality, dynamic performance and parameter sensitivity, enable a quantitative comparison of the four controllers. The indicators are not only obtained from simulation results, they are also experimentally validated on a $4 \mathrm{~kW}$ axial flux permanent magnet synchronous machine with yokeless and segmented armature topology. A general conclusion is that the PI controller excels when it comes to control quality: only the deadbeat controller can compete with its low torque ripple. The predictive controllers on the other hand show superior dynamic performance.
\end{abstract} trol

Index Terms-Permanent magnet machines, predictive con-

\section{INTRODUCTION}

The continuous evaluation of digital signal processing in the last half-century offered immense potential for converter control techniques. Besides the already existing analog controllers - such as the commonly used PI controllers - new, more advanced control techniques could be developed, in order to improve the transient performance and efficiency of electric drives. The emergence of fast control hardware platforms like field programmable gate arrays (FPGAs), for instance, made predictive control a full-fledged alternative for control of electric drives with time constants in the millisecond range [1].

A predictive control strategy that is frequently mentioned in the context of inverter fed ac machines - and is the first predictive control scheme that will be implemented in this article - is finite-set model based predictive control (FSMBPC) [1]-[5]. It makes use of a process model to forecast the future behavior for each of the steering actions, and selects the most optimal one based on the evaluation of a cost function. Such steering actions in case of a two-level voltage source inverter (2L-VSI) are its eight possible switch

The authors L. Verkroost, J. Druant, H. Vansompel, F. De Belie, and P. Sergeant are with the Electrical Energy Laboratory, Department of Electrical Energy, Metals, Mechanical Constructions and Systems, Ghent University, 9000 Ghent, Belgium, and with EEDT, Flanders Make, Flanders, Belgium (e-mail: Lynn. Verkroost@UGent.be, Joachim.Druant@UGent.be; Hendrik.Vansompel@UGent.be; $\quad$ Frederik.DeBelie@UGent.be; Peter.Sergeant@UGent.be).

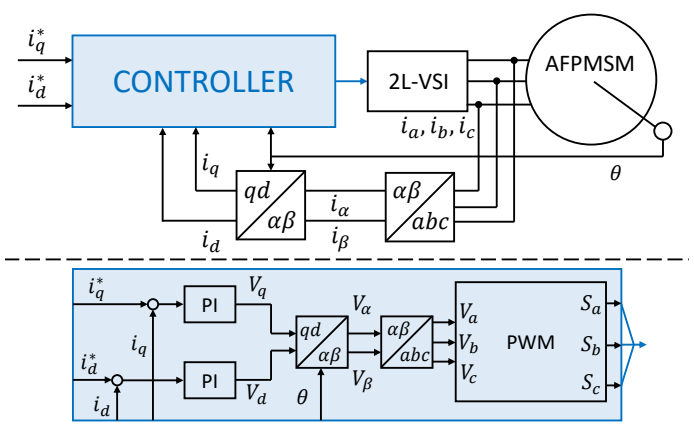

(a)

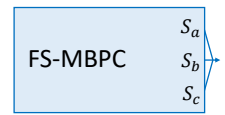

(b)

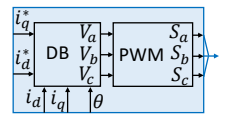

(c)

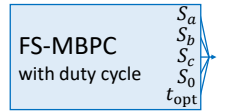

(d)
Fig. 1. General control scheme, with the four examined controllers: (a) PI control, (b) FS-MBPC, (c) DB control, (d) FS-MBPC with duty cycle calculation

states. Due to the flexibility of FS-MBPC in the type of system model and cost function to be used, this technique is very well-suited to control multiple-input multiple-output (MIMO) systems, and to deal with various non-linearities and constraints. Some of its major drawbacks, however, are its high computational burden required to evaluate all the feasible inputs, its variable switching frequency $f_{\text {switch }}$ making filter design more complicated, and the large torque ripple due to the pulsed voltage input. A technique to relieve the calculation effort of FS-MBPC is reducing the amount of voltage vectors that needs to be evaluated, and is presented in [3]. The issue of a variable $f_{\text {switch }}$ and high torque ripple is resolved in [6]. However, the method proposed in [6] requires the solution of a quadratic optimization problem, abolishing the simplicity of standard FS-MBPC.

A second type of predictive controller that is included in the comparative study - called deadbeat (DB) control solves the same issues of an unacceptably high torque ripple and a variable $f_{\text {switch. }}$. The basic principle comprises the calculation of the required input voltage to reduce the error on the controlled variables to zero in a finite amount of steps (preferably one). A requirement for DB control is pulse width modulation (PWM). Similar methods are used in [3], [7], [8] to implement predictive torque control. Unfortunately, DB control does not make use of a cost function. As a result, the flexibility to impose various constraints by adapting such a 
cost function is lost in this approach.

Therefore, a third predictive control algorithm that will be discussed and compared in this article is a hybrid version of the two previous controllers, and is proposed in [2] for a radial permanent magnet synchronous machine (PMSM), and in [9], [10] for an induction motor. The working principle is the same as for standard FS-MBPC, with the only difference that the DB principle is used to determine an appropriate duty cycle for each active voltage vector of the 2L-VSI. In this way, the torque ripple is reduced and a fixed $f_{\text {switch }}$ is ensured, while the ability to express secondary control goals is maintained. The technique proposed in [11] for a matrix converter and in [12] for a 2L-VSI is based on a similar principle: the controller determines for each update period an optimal sequence of voltage inputs by means of a cost function.

Since the PI controller is still one of the most frequently used controllers in industry [13], the aim of this article is to compare the more advanced and more complex predictive controllers not only with each other, but also with a standard PI controller. Although comparative studies between two or more predictive controllers, or between a certain type of predictive controller and a PI controller already exist in literature [2], [3], [6], [7], [9], [10], such a global comparison of the three above-mentioned predictive controllers and a PI controller has never been performed. In this article, the update frequency $\left(f_{\mathrm{u}}\right)$ is chosen to be equal for all the control algorithms (instead of a constant $f_{\text {switch }}$ ), in order to provide a common basis for the calculation time of the controllers. Due to the improvements in the performance of the FPGAs in the last decades, it is not the controller but the inverter that imposes limitations on $f_{\mathrm{u}}$ in the considered test setup. Therefore the timing of the sampling is adapted in order to obtain a more accurate linearization of the model. Another important aspect is that all the obtained simulation results are experimentally verified on a $4 \mathrm{~kW}$ prototype of an axial flux permanent magnet synchronous machine (AFPMSM) with surface mounted permanent magnets (PMs), a yokeless and segmented armature (YASA) topology and fractional slot windings [14], fed by a 2L-VSI. The comparative study covers both steady-state and transient operation.

\section{Machine Model Description}

Since an AFPMSM possesses no rotor windings, the dynamic electrical equations only need to describe the dynamic operation of the stator windings. The discrete state-space model of the machine in the synchronous $q d$-reference frame with time step $\Delta t$ is expressed as:

$$
\begin{array}{r}
{\left[\begin{array}{l}
i_{q}^{k+1} \\
i_{d}^{k+1}
\end{array}\right]=\left[\begin{array}{cc}
1-\frac{R_{\mathrm{s}} \Delta t}{L_{q}} & \omega^{k} \Delta t \\
-\omega^{k} \Delta t & 1-\frac{R_{\mathrm{s}} \Delta t}{L_{d}}
\end{array}\right]\left[\begin{array}{c}
i_{q}^{k} \\
i_{d}^{k}
\end{array}\right]+\left[\begin{array}{cc}
\frac{\Delta t}{L_{q}} & 0 \\
0 & \frac{\Delta t}{L_{d}}
\end{array}\right]\left[\begin{array}{c}
v_{q}^{k} \\
v_{d}^{k}
\end{array}\right]} \\
+\left[\begin{array}{c}
\omega^{k} i_{\operatorname{mag}} \Delta t \\
0
\end{array}\right] .
\end{array}
$$

The involved machine parameters are tabulated in Table I. Since the considered machine has surface mounted PMs,
TABLE I

MACHINE PARAMETERS

\begin{tabular}{lll}
\hline \hline Parameter & Symbol & Value \\
\hline Number of pole pairs & $N_{p}$ & 8 \\
Rated power $(\mathrm{kW})$ & $P_{\mathrm{n}}$ & 4 \\
Rated speed $(\mathrm{rpm})$ & $N_{\mathrm{n}}$ & 2500 \\
Rated torque $(\mathrm{Nm})$ & $T_{\mathrm{n}}$ & 15 \\
Rated voltage $(\mathrm{V})$ & $V_{\mathrm{n}}$ & 152 \\
Stator inductance $(\mathrm{mH})$ & $L_{q}=L_{d}$ & 2.54 \\
Stator resistance $(\mathrm{m} \Omega)$ & $R_{\mathrm{S}}$ & 325 \\
Mechanical inertia $\left(\mathrm{kg} \cdot \mathrm{m}^{2}\right)$ & $J$ & 0.0024 \\
Equivalent PM current $(\mathrm{A})$ & $i_{\mathrm{mag}}$ & -43.2 \\
\hline
\end{tabular}

the stator inductances $L_{q}$ and $L_{d}$ are equal. $\omega^{k}(\mathrm{rad} / \mathrm{s})$ represents the electrical speed at discrete time instant $k ; i_{\text {mag }}$ is a constant equivalent current along the negative $d$-axis, representing the PMs and resulting in the same flux level as generated by the PMs.

The developed electromagnetic torque $T_{\mathrm{em}}$ is directly related to $i_{q}$ :

$$
T_{\mathrm{em}}^{k}=-\frac{3}{2} N_{p} L_{d} i_{\mathrm{mag}} i_{q}^{k}
$$

Hence, a reference value $i_{q}^{*}$ can be used to substitute a reference value $T_{\mathrm{em}}^{*}$. For surface-mounted PMSMs, minimization of the copper losses is obtained by fixing $i_{d}^{*}$ to zero.

\section{Predictive Control Schemes}

\section{A. Finite-Set Model Based Predictive Control}

The control scheme of FS-MBPC is presented in Fig. 1b. Its working principle is illustrated in Fig. 2 and is discussed in detail in [5]. Three important steps can be defined.

1) Estimation: Based on knowledge of the optimal control action $S_{k}$ (the inverter's switch state determined during the previous time interval $k-1 \rightarrow k$ and applied during the interval $k \rightarrow k+1$ ), and measurements of the stator current and the rotor position at instant $k+1 / 2$, the stator current components $i_{q}^{k+1}$ and $i_{d}^{k+1}$ at instant $k+1$ can be estimated by means of the machine and inverter model provided in Section II (with $\Delta t=T_{\mathrm{u}} / 2$ ). The state is measured half an update period after $S_{k}$ is applied in order to avoid measuring transients. Moreover, the time step used in the discrete time model is halved by sampling at instant $k+1 / 2$ instead of $k$, leading to a more accurate discretized model. Since it is not the controller but the inverter that imposes limitations on $f_{\mathrm{u}}$ in the specific test setup used in this article, halving the computation time of the microcontroller poses no obstacle.

The estimation step is included, since knowledge of the state variables at instant $k+1$ is required to find the optimal input at instant $k+1$. Measuring this state is not an option, as this would mean that the controller should be able to calculate $S_{k+1}$ in an infinitely small period of time.

2) Prediction: For each of the eight possible switch states $S_{k+1}$, the controlled variables $i_{q}^{k+2}$ and $i_{d}^{k+2}$ at time instant $k+2$ are predicted by means of the system model (1) (with $\Delta t=T_{\mathrm{u}}$ ), starting from the estimates $\hat{i}_{q}^{k+1}$ and $\hat{i}_{d}^{k+1}$. 


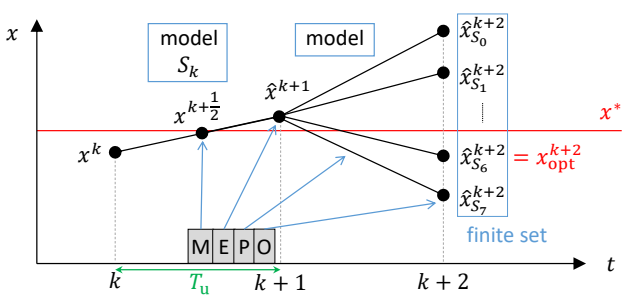

Fig. 2. Working principle of FS-MBPC, visualizing the basic steps: measurement $(\mathrm{M})$, estimation $(\mathrm{E})$, prediction $(\mathrm{P})$, and optimization $(\mathrm{O})$

3) Optimization: Based on the evaluation of a cost function, the most appropriate switch state is selected, bringing the system state closest to its desired value $\left[i_{q}^{*}, i_{d}^{*}\right]$. This optimal switch state $S_{k+1}$ is applied to the system at instant $k+1$ (and kept constant during the interval $k+1 \rightarrow k+2$, as is illustrated in Fig. 4a), after which the algorithm is restarted. This is the so-called receding horizon principle. It is noteworthy that only the eight voltage vectors according to the eight different switch states of the 2L-VSI can be applied to the AFPMSM during $T_{\mathrm{u}}$, yielding a high current ripple.

The cost function can be defined in various ways. A common choice is to express it as the sum of the squares of the deviations of the controlled variables from their setpoints:

$$
J^{k+1}=\left(\hat{i}_{q}^{k+2}-i_{q}^{*}\right)^{2}+W_{I}\left(\hat{i}_{d}^{k+2}-i_{d}^{*}\right)^{2},
$$

where $W_{I}$ is a dimensionless weighting factor representing the relative strictness of the control of the current components. This factor needs to be fine-tuned [1], [4]. In this paper, $W_{I}=1$. Secondary control goals, such as reduction of the switching losses, or safety limits can be expressed easily by adding extra cost terms [1], [3], [6].

\section{B. Deadbeat Control}

The control scheme and working principle of DB control are illustrated in Fig. 1c and 3 respectively. Contrary to FSMBPC, DB control comprises only two major steps.

1) Estimation: The required input voltage $V_{k}^{*}$, applied to the machine at instant $k$, and the measured $i_{q}^{k+1 / 2}, i_{d}^{k+1 / 2}$ and $\omega^{k+1 / 2}$ are used to calculate the system state at instant $k+1$ by means of the system model provided in Section II (with $\Delta t=T_{\mathrm{u}} / 2$ ).

2) Deadbeat: To obtain the voltage $V_{k+1}^{*}$, required to bring $i_{q}^{k+2}$ and $i_{d}^{k+2}$ to their respective reference values $i_{q}^{*}$ and $i_{d}^{*}$ in one time step $T_{\mathrm{u}}$, it is assumed that the stator current prediction equals its setpoint at instant $k+2: \hat{i}_{q}^{k+2}=i_{q}^{*}$ and $\hat{i}_{d}^{k+2}=i_{d}^{*}$. A model inverse solution, based on (1), is then used to compute the voltage components $v_{q}^{k+1}$ and $v_{d}^{k+1}$ to be applied at $k+1$.

The reference voltages $v_{x \mathrm{o}}(x \in\{a, b, c\})$ are obtained by applying the inverse Park transformation. Eventually, a PWM algorithm determines the sequence of switch states that needs to be applied during $T_{\mathrm{u}}$. Since PWM enables to vary the duty cycle of each inverter leg separately - as is illustrated in Fig. $4 \mathrm{~b}$ - both the magnitude and the direction of the on average applied voltage vector become variable.

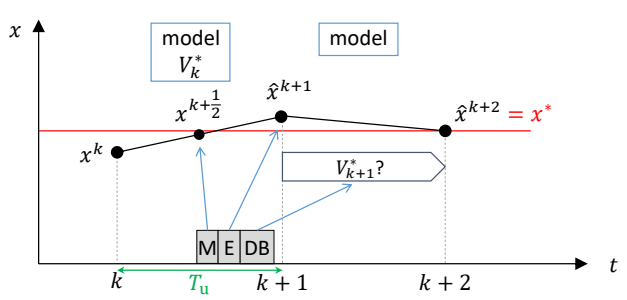

Fig. 3. Working principle of DB control, visualizing the basic steps: measurement $(\mathrm{M})$, estimation $(\mathrm{E})$, and deadbeat (DB)

\section{FS-MBPC with Duty Cycle Calculation}

Fig. 1d shows the control scheme for FS-MBPC with duty cycle calculation. Its structure is similar to that of standard FS-MBPC. Three important steps can be defined in the working principle.

1) Estimation: This step is identical to the estimation step of FS-MBPC.

2) Prediction: Contrary to standard FS-MBPC, only the six active voltage vectors of the 2L-VSI are evaluated separately. Just a fraction of the control period is allocated to this active voltage vector, the rest of the time a null voltage is applied. Therefore, two slopes must be calculated for each of the six active voltage vectors: the slope of the current for the fraction of the period in which the active vector is applied (denoted as $s_{i}(i=1 \ldots 6)$ ), and the slope for when the zero vector is applied (denoted as $s_{0}$ ). Those slopes can be calculated by means of the dynamical equations (1).

$$
\left\{\begin{array}{l}
s_{q, 0}^{k+1}=\frac{1}{L_{q}}\left[-R_{\mathrm{s}} \hat{i}_{q}^{k+1}+\omega^{k+1 / 2} L_{d}\left(\hat{i}_{d}^{k+1}+i_{\mathrm{mag}}\right)\right] \\
s_{q, i}^{k+1}=\frac{1}{L_{q}}\left[v_{q, i}^{k+1}-R_{\mathrm{s}} \hat{i}_{q}^{k+1}+\omega^{k+1 / 2} L_{d}\left(\hat{i}_{d}^{k+1}+i_{\mathrm{mag}}\right)\right]
\end{array}\right.
$$

$$
\left\{\begin{array}{l}
s_{d, 0}^{k+1}=\frac{1}{L_{d}}\left[-R_{\mathrm{s}} \hat{i}_{d}^{k+1}-\omega^{k+1 / 2} L_{q} \hat{i}_{q}^{k+1}\right] \\
s_{d, i}^{k+1}=\frac{1}{L_{d}}\left[v_{d, i}^{k+1}-R_{\mathrm{s}} \hat{i}_{d}^{k+1}-\omega^{k+1 / 2} L_{q} \hat{i}_{q}^{k+1}\right]
\end{array}\right.
$$

The optimal duration $t_{\mathrm{opt}, i}^{k+1}$ for the active voltage vector under consideration is determined according to the deadbeat principle:

$$
i_{q}^{*}=\hat{i}_{q}^{k+2}=\hat{i}_{q}^{k+1}+s_{q, 0}^{k+1}\left(T_{\mathrm{u}}-t_{\mathrm{opt}, i}^{*}\right)+s_{q, i}^{k+1} t_{\mathrm{opt}, i}^{*},
$$

and consequently:

$$
\begin{gathered}
t_{\mathrm{opt}, i}^{*}=\frac{i_{q}^{*}-\hat{i}_{q}^{k+1}-s_{q, 0}^{k+1} T_{\mathrm{u}}}{s_{q, i}^{k+1}-s_{q, 0}^{k+1}} \\
\begin{cases}t_{\mathrm{opt}, i}^{k+1}=0, & \text { if } t_{\mathrm{opt}, i}^{*}<0 \\
t_{\mathrm{opt}, i}^{k+1}=t_{\mathrm{opt}, i}^{*}, & \text { if } 0 \leq t_{\mathrm{opt}, i}^{*} \leq T_{\mathrm{u}} \\
t_{\mathrm{opt}, i}^{k+1}=T_{\mathrm{u}}, & \text { if } T_{\mathrm{u}}<t_{\mathrm{opt}, i}^{*} .\end{cases}
\end{gathered}
$$

Eventually, the current components at instant $k+2$ can be predicted based on $s_{0}^{k+1}, s_{i}^{k+1}$ and $t_{\mathrm{opt}, i}^{k+1}$.

$$
\begin{array}{r}
\hat{i}_{q}^{k+2}=\hat{i}_{q}^{k+1}+s_{q, 0}^{k+1}\left(T_{\mathrm{u}}-t_{\mathrm{opt}, i}^{k+1}\right)+s_{q, i}^{k+1} t_{\mathrm{opt}, i}^{k+1} \\
\hat{i}_{d}^{k+2}=\hat{i}_{d}^{k+1}+s_{d, 0}^{k+1}\left(T_{\mathrm{u}}-t_{\mathrm{opt}, i}^{k+1}\right)+s_{d, i}^{k+1} t_{\mathrm{opt}, i}^{k+1}
\end{array}
$$




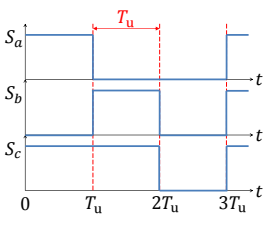

(a)

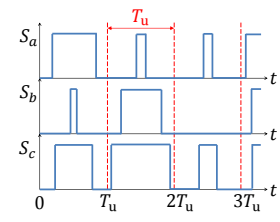

(b)

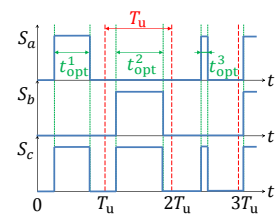

(c)
Fig. 4. Difference in switching between (a) FS-MBPC, (b) PI and DB control, (c) FS-MBPC with duty cycle calculation

3) Optimization: This step is identical to the optimization step of FS-MBPC. The selected null vector ([ $\left.\begin{array}{lll}0 & 0 & 0\end{array}\right]$ or $\left.\left[\begin{array}{lll}1 & 1 & 1\end{array}\right]\right)$ is the one that requires the lowest number of switches to change their state. Contrary to DB control followed by PWM, the optimization of the duty cycle only allows to change the magnitude of the applied voltage vector, and not its direction. The difference in switching is illustrated in Fig. 4.

\section{Simulation And Experimental Results}

To compare the performance of the predictive controllers, they are first simulated in a MATLAB $^{\circledR} \&$ Simulink $\left.{ }^{(}\right)$environment. A PI controller with anti-windup and parameters $K_{p}=4.13$ and $K_{i}=3206.4$ is included to provide a benchmark for the comparison of the predictive controllers. Its control scheme is presented in Fig. 1a. Afterwards, their real-life performance is validated by conducting the same tests as in simulation on a $4 \mathrm{~kW}$ test setup. A DCbus voltage $V_{\mathrm{dc}}$ of $250 \mathrm{~V}$ is applied, and the mechanical speed of the machine is maintained at $1000 \mathrm{rpm}$. The chosen $f_{\mathrm{u}}$ amounts to $10 \mathrm{kHz}$. The specifications of the AFPMSM - designed according to the principles proposed in [14] - are given in Table I. The control algorithms are implemented on a Xilinx ${ }^{\complement}$ Kintex $^{\complement}{ }_{-7}$ XC7K325T FPGA embedded in a dSPACE MicroLabBox. The FPGA is programmed using the Xilinx System Generator blockset in a MATLAB ${ }^{\complement}$ \&Simulink $^{\complement}$ environment. The AFPMSM is connected to an induction machine (controlled by means of a DS1104 R\&D Controller Board of dSPACE), ensuring the constant speed of the AFPMSM.

The simulation results in Fig. 5 show that all controllers are able to track their references. Fig. 7 indicates that all controllers follow $i_{q}^{*}$ in real-life as well. However, due to model inaccuracy, $i_{d}$ deviates from zero for the predictive controllers, causing an increase in the copper losses. The following paragraphs describe the simulation results of Fig. 5 and the experimental results of Fig. 7 in detail.

\section{A. Control Quality}

The difference in current ripple - and thus torque ripple - strikes immediately in Fig. 5 and 7. As the current ripple causes high frequency components of the magnetic flux density in the permanent magnets and in the stator core laminations, a high ripple results in high eddy current losses in the permanent magnets and additional stator core

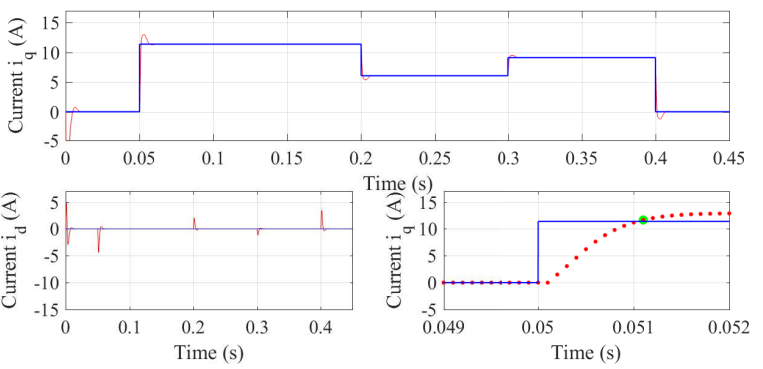

(a) PI control

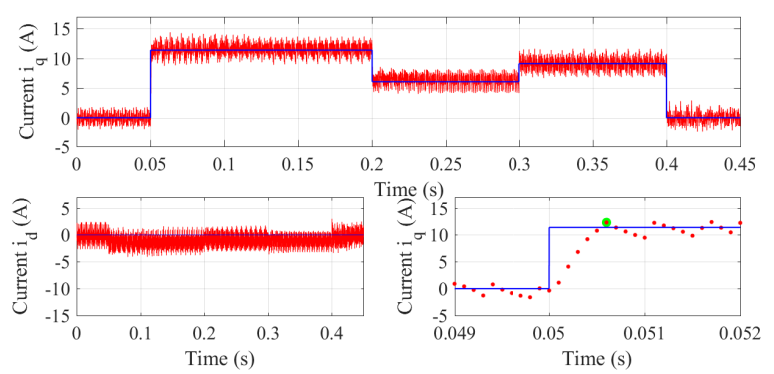

(b) FS-MBPC

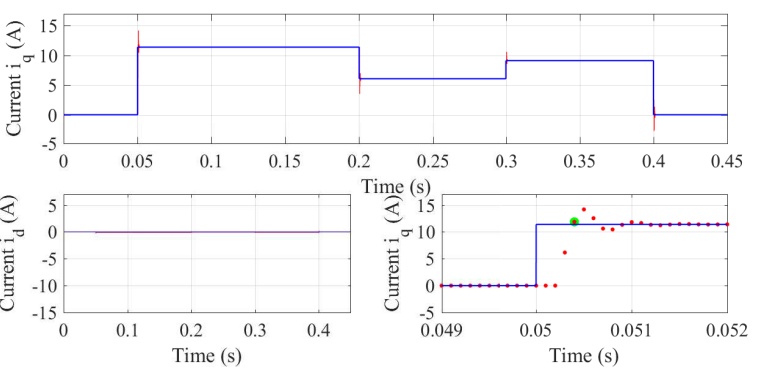

(c) DB control

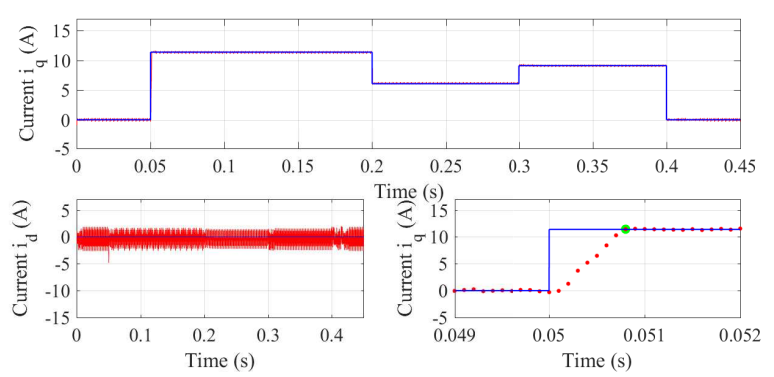

(d) FS-MBPC with duty cycle calculation

Fig. 5. Simulation results for the stator current components (red: simulation, blue: reference)
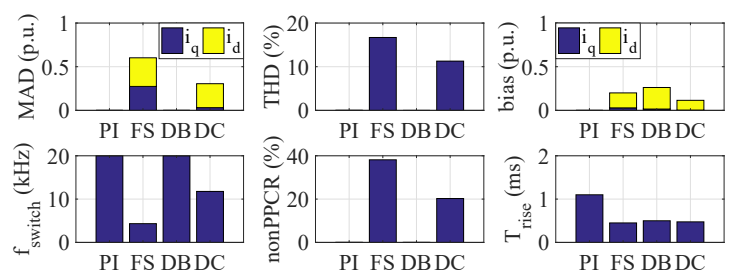

Fig. 6. Simulated key performance indicators for PI control, FS-MBPC (FS), DB control and FS-MBPC with duty cycle calculation (DC) 


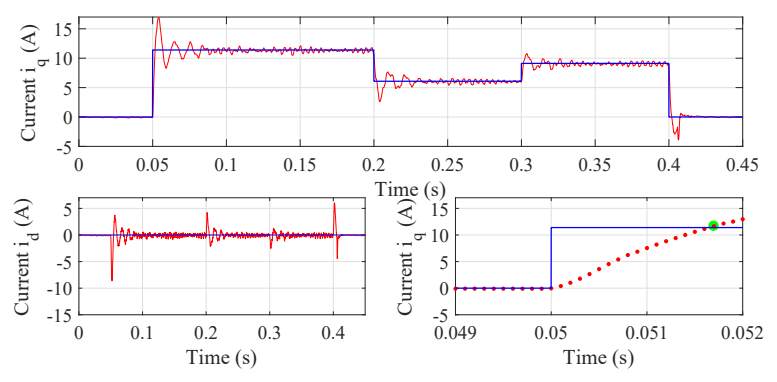

(a) PI control

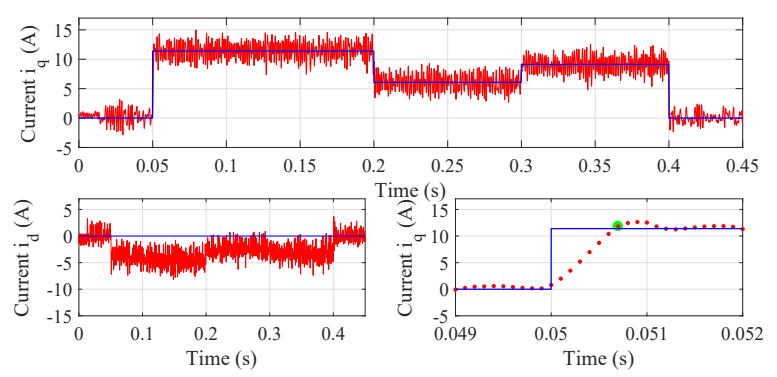

(b) FS-MBPC

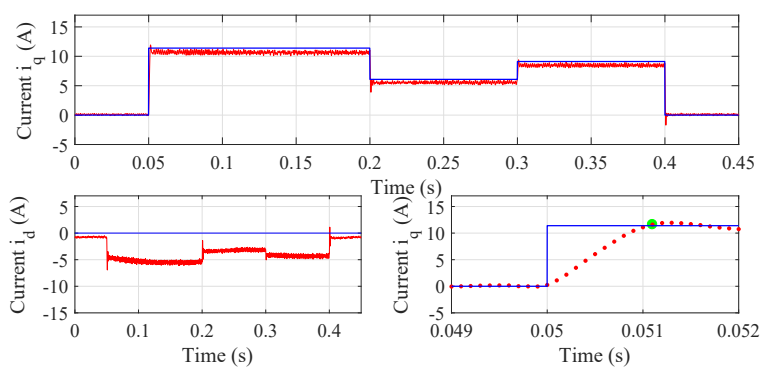

(c) DB control

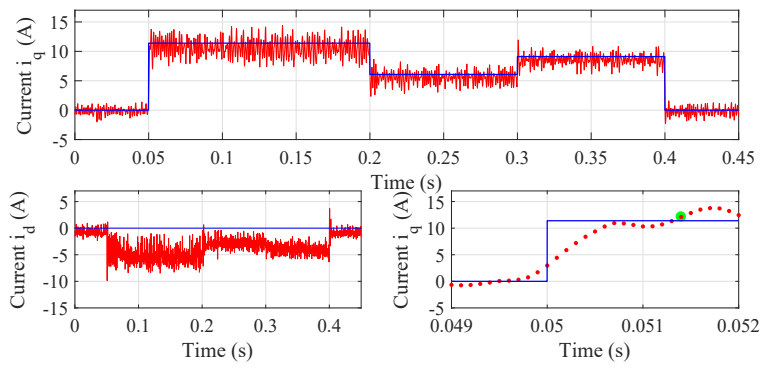

(d) FS-MBPC with duty cycle calculation

Fig. 7. Experimental results for the stator current components (red: measurement, blue: reference)

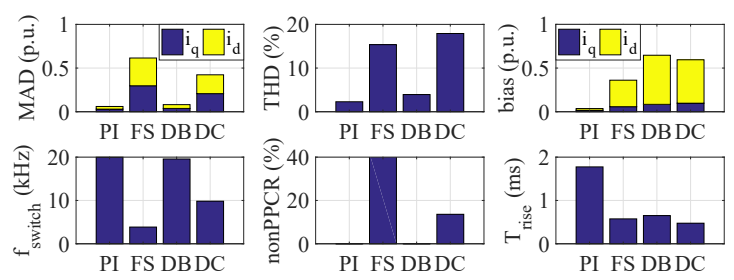

Fig. 8. Experimental key performance indicators losses. To quantify the ripple caused by the controllers, the mean absolute deviation (MAD) will be used throughout this article.

$$
\operatorname{MAD}(x)=\sum_{k=1}^{m}\left|\frac{\bar{x}-x_{k}}{m x_{k}^{*}}\right|
$$

$m$ is the amount of samples used in the calculation, $\bar{x}$ is the average value of the controlled variable $x$ over these $m$ samples, and $x_{k}^{*}$ is its setpoint (unless the setpoint is zero, then $x_{k}^{*}=1$ ). The MADs shown in Fig. 6 and 8 for the simulations and the experiments respectively, reveal the superior performance of the PI and DB controller concerning current ripple. The simulations show an invisibly small current ripple for the PI and DB controller. The measurements for standard FS-MBPC display a ripple that is ten times higher in magnitude for both $i_{q}$ and $i_{d}$ compared to the PI controller. Adding the calculation of $t_{\mathrm{opt}}$ to the FS-MBPC algorithm reduces the average amplitude of the ripple in $i_{q}$ - and thus the ripple in the torque - with a factor six in simulation. The effect on the ripple in $i_{d}$ is less pronounced (a reduction with a factor 1.6), since the duty cycle calculation focusses on $i_{q}$ and not on $i_{d}$. In reality, the effect of the duty cycle calculation is slightly disappointing: although the average current ripple reduces for both current components to only a factor seven higher than for the PI controller in the experimental results, its maximal amplitude is still as high as for standard FS-MBPC. Moreover, the experimental results in Fig. 8 indicate that the total harmonic distortion (THD) of the stator current (calculated up to the 1000th-order harmonic) slightly increases by adding the duty cycle calculation to FSMBPC, although this is not expected from the simulation results in Fig. 6.

Since the main goal of the controllers is to track the reference values for $i_{q}$ and $i_{d}$ precisely, also the average deviation of these controlled variables from their setpoints $i_{q}^{*}$ and $i_{d}^{*}$ is of importance. This so-called bias is calculated as:

$$
\operatorname{bias}(x)=\left|\sum_{k=1}^{m} \frac{x_{k}-x_{k}^{*}}{m x_{k}^{*}}\right|
$$

and is most pronounced for $i_{d}$. Both the simulations and the experiments show that only the predictive controllers, and especially the DB controller, suffer from this disadvantage. The bias originates from the small prediction errors due to the discretization of the machine model in the simulation results, whereas model inaccuracy comes into play for the experiments as well. In simulation, the DB controller exhibits more than three times the bias of FS-MBPC with duty cycle calculation and 1.2 times the bias of the standard FS-MBPC. The experiments give somewhat different results: the bias values for DB control and FS-MBPC with duty cycle calculation are of similar magnitude, while the bias for standard FS-MBPC is $44 \%$ lower than for DB control. Many methods have been proposed in order to improve the robustness against model mismatch for predictive control, and especially for DB control. The authors of [15], for instance, enhanced the robustness of DB control against parameter uncertainties by 
adding a discrete-time integral term to the prediction step. In [16], a recursive least squares algorithm was used to estimate the machine parameters online. The methods proposed in [17], [18] ameliorated standard DB control by making use of observers to eliminate the influence of parameter mismatch online. However, none of these methods is applied in this article.

\section{B. Switching Frequency and Pulse Polarity Consistency Rule}

Standard FS-MBPC excels when it comes to switching losses. Since standard FS-MBPC does not allow the switch state to be changed during $T_{\mathrm{u}}$ - while this is possible for the other three controllers, as is illustrated in Fig. 4 - its switching frequency $f_{\text {switch }}$ (defined as the number of times a switch changes its state from on to off or vice-versa, averaged over a certain period of time and the three pairs of complementary switches of the 2L-VSI) will always be lower than $f_{\mathrm{u}}$ $\left(f_{\mathrm{u}}=10 \mathrm{kHz}\right)$. In [2] is verified that FS-MBPC leads to an unpredictable, variable switching frequency, depending on the operating point. In this particular case, $f_{\text {switch }}=40 \% f_{\mathrm{u}}$ both in simulation and in reality, as can be seen in Fig. 6 and 8. Since PWM causes each switch to change its state twice per $T_{\mathrm{u}}$, a general conclusion is that $f_{\text {switch }} \approx 2 \cdot f_{\mathrm{u}}$ for both the PI and DB controller. For FS-MBPC with duty cycle calculation, the average $f_{\text {switch }}$ can be estimated. When the null vector equals [ $\left[\begin{array}{lll}0 & 0 & 0\end{array}\right]$, for instance, there are three active voltage vectors that only require one inverter leg to change its switch state twice, and three active voltage vectors that require two inverter legs to change their switch state twice. Therefore each leg switches once per $T_{\mathrm{u}}$ on average, and thus $f_{\text {switch }} \approx f_{\mathrm{u}}$.

Although standard FS-MBPC requires the lowest $f_{\text {switch }}$, the switch state changes that do take place violate the pulse polarity consistency rule (PPCR) in $40 \%$ of the cases, hence heavily burdening the machine isolation. In Fig. 6 and 8, both the simulations and experiments indicate that the higher $f_{\text {switch }}$ when duty cycle calculation is added causes the PPCR to be fulfilled more often. For DB and PI control, almost no violations of the PPCR take place anymore.

\section{Dynamic Behavior}

As to dynamic behavior, only the DB and PI controller show distinct overshoot peaks in $i_{q}$ when a step is applied in their reference value. For the first step in $i_{q}^{*}$, the rise time of the controllers can be noticed on the zoomed-in views for $i_{q}$ in Fig. 5 and 7. The values for the rise time mentioned in Fig. 6 and 8 are obtained by averaging the rise times for all the steps applied in $i_{q}^{*}$. This averaged rise time required to bring $i_{q}$ to its reference value is significantly higher for the PI controller than for the predictive controllers: $1.1 \mathrm{~ms}$ for the PI controller against maximum $0.5 \mathrm{~ms}$ for the predictive controllers in simulation, and $1.8 \mathrm{~ms}$ against maximum $0.7 \mathrm{~ms}$ in reality. In other words: the predictive controllers outshine the PI controller when it comes to dynamic performance.

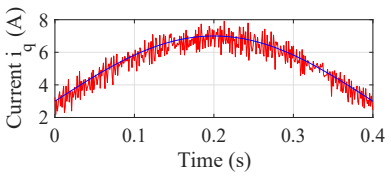

(a) FS-MBPC

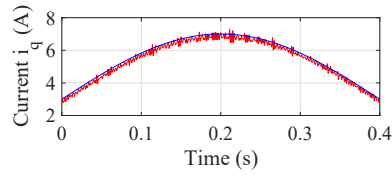

(b) FS-MBPC with duty cycle
Fig. 9. Experimental results for $i_{q}$ under $V_{\mathrm{dc}}=100 \mathrm{~V}$ and $N=300 \mathrm{rpm}$

\section{Influence of the Working Conditions}

The foregoing comparison is based on the experimental results of Fig. 7, for DC-bus voltage $V_{\mathrm{dc}}=250 \mathrm{~V}$ and mechanical speed $N=1000 \mathrm{rpm}$. Under these working conditions, the effect of adding the duty cycle calculation to standard FS-MBPC is slightly disappointing: it only reduces the average current ripple, although it was expected to reduce the maximal ripple amplitude as well. The reason for this phenomenon is the fact that for $N=1000 \mathrm{rpm}$, $V_{\mathrm{dc}}=250 \mathrm{~V}$ is just sufficient to surpass the back-emf. As a result, the optimal duty cycle equals 0 or $T_{\mathrm{u}}$ at certain instants, leading to the same ripple amplitude as for standard FS-MBPC. However, when working under $V_{\mathrm{dc}}=100 \mathrm{~V}$ and $N=300 \mathrm{rpm}$, this is not the case anymore, and addition of the duty cycle does reduce the maximal ripple amplitude as well, as can be seen by comparing Fig. 9a and 9b.

\section{E. PI Controller versus Predictive Controllers}

It is very striking that the PI controller often outperforms the more advanced predictive controllers in the foregoing discussion. The main reason for this outcome, is the fact that the major strengths of the predictive controllers are not fully exploited by the application under consideration. FSMBPC, for instance, is particularly well-suited to deal with MIMO and highly non-linear systems. In addition, this type of controller is well-known for its natural way of dealing with several constraints. An AFPMSM fed by a 2L-VSI does not make an appeal to any of these strengths.

\section{CONCLUSion}

Three different predictive current controllers and a PI controller have been compared both in simulation and in experiments. For this purpose, some key performance indicators were introduced. The three predictive controllers were FS-MBPC, DB control and FS-MBPC with duty cycle calculation, and the studied drive was an AFPMSM with surface mounted permanent magnets and a YASA-topology, fed by a 2 L-VSI. The major disadvantages of FS-MBPC that emerged are its high torque and current ripple, its high harmonic content in the stator current, and its poor performance concerning the fulfillment of the PPCR. However, this controller excels in its low switching losses. The DB and PI controller on the contrary, show outstanding results concerning current ripple, THD and PPCR, but they lead to high switching losses and exhibit distinct overshoot peaks. The major weakness of the DB controller is its systematic error 
in both current components. The most important drawback of the PI controller compared to the predictive controllers is its relatively long rise time. Since FS-MBPC with duty cycle calculation combines the principles of both DB control and FS-MBPC, it also combines the performance features of those control algorithms. Overall, the PI controller outhweighs the predictive controllers concerning control quality, whereas the predictive controllers excel in dynamic performance.

\section{REFERENCES}

[1] T. J. Vyncke, S. Thielemans, and J. A. Melkebeek, "Finite-set modelbased predictive control for flying-capacitor converters: Cost function design and efficient FPGA implementation," IEEE Trans. Ind. Informat., vol. 9, no. 2, pp. 1113-1121, May 2013.

[2] F. Morel, X. Lin-Shi, J. M. Retif, B. Allard, and C. Buttay, "A comparative study of predictive current control schemes for a permanentmagnet synchronous machine drive," IEEE Trans. Ind. Electron., vol. 56, no. 7, pp. 2715-2728, Jul. 2009.

[3] W. Xie, X. Wang, F. Wang, W. Xu, R. M. Kennel, D. Gerling, and R. D. Lorenz, "Finite-control-set model predictive torque control with a deadbeat solution for pmsm drives," IEEE Trans. Ind. Electron., vol. 62, no. 9, pp. 5402-5410, Sep. 2015.

[4] T. Vyncke, S. Thielemans, M. Jacxsens, and J. Melkebeek, "Analysis of some design choices in model based predictive control of flyingcapacitor inverters," COMPEL-The international journal for computation and mathematics in electrical and electronic engineering, vol. 31, no. 2, pp. 619-635, 2012.

[5] S. Kouro, P. Cortes, R. Vargas, U. Ammann, and J. Rodriguez, "Model predictive control - a simple and powerful method to control power converters," IEEE Trans. Ind. Electron., vol. 56, no. 6, pp. 1826-1838, Jun. 2009.

[6] A. D. Alexandrou, N. K. Adamopoulos, and A. G. Kladas, "Development of a constant switching frequency deadbeat predictive control technique for field-oriented synchronous permanent-magnet motor drive," IEEE Trans. Ind. Electron., vol. 63, no. 8, pp. 5167-5175, Aug. 2016.

[7] H. Zhu, X. Xiao, and Y. Li, "Torque ripple reduction of the torque predictive control scheme for permanent-magnet synchronous motors," IEEE Trans. Ind. Electron., vol. 59, no. 2, pp. 871-877, Feb. 2012.

[8] B. H. Kenny and R. D. Lorenz, "Stator and rotor flux based deadbeat direct torque control of induction machines," in Proc. Conf. Record of the 2001 IEEE Industry Applications Conf.. 36th IAS Annual Meeting (Cat. No.01CH37248), vol. 1, Sep. 2001, pp. 133-139 vol.1.

[9] Y. Zhang and H. Yang, "Model predictive torque control of induction motor drives with optimal duty cycle control," IEEE Transactions on Power Electronics, vol. 29, no. 12, pp. 6593-6603, Dec. 2014.

[10] M. Nemec, D. Nedeljkovic, and V. Ambrozic, "Predictive torque control of induction machines using immediate flux control," IEEE Trans. Ind. Electron., vol. 54, no. 4, pp. 2009-2017, Aug. 2007.

[11] M. Vijayagopal, P. Zanchetta, L. Empringham, L. de Lillo, L. Tarisciotti, and P. Wheeler, "Control of a direct matrix converter with modulated model-predictive control," IEEE Trans. Ind. Appl., vol. 53, no. 3, pp. 2342-2349, 2017.

[12] M. Rivera, F. Morales, C. Baier, J. Muoz, L. Tarisciotti, P. Zanchetta, and P. Wheeler, "A modulated model predictive control scheme for a two-level voltage source inverter," in Proc. IEEE Int. Conf. Industrial Technology (ICIT), Mar. 2015, pp. 2224-2229.

[13] H. Wu, W. Su, and Z. Liu, "PID controllers: Design and tuning methods," in Proc. 9th IEEE Conf. Industrial Electronics and Applications, Jun. 2014, pp. 808-813.

[14] H. Vansompel, P. Sergeant, L. Dupr, and A. V. den Bossche, "Axialflux pm machines with variable air gap," IEEE Trans. Ind. Electron., vol. 61, no. 2, pp. 730-737, Feb. 2014.

[15] T. Turker, U. Buyukkeles, and A. F. Bakan, "A robust predictive current controller for pmsm drives," IEEE Trans. Ind. Electron., vol. 63, no. 6, pp. 3906-3914, Jun. 2016.

[16] S. J. Underwood and I. Husain, "Online parameter estimation and adaptive control of permanent-magnet synchronous machines," IEEE Trans. Ind. Electron., vol. 57, no. 7, pp. 2435-2443, Jul. 2010.
[17] X. Zhang, B. Hou, and Y. Mei, "Deadbeat predictive current control of permanent-magnet synchronous motors with stator current and disturbance observer," IEEE Trans. Power Electron., vol. 32, no. 5, pp. 3818-3834, May 2017.

[18] C. Zhang, G. Wu, F. Rong, J. Feng, L. Jia, J. He, and S. Huang, "Robust fault-tolerant predictive current control for permanent magnet synchronous motors considering demagnetization fault," IEEE Trans. Ind. Electron., vol. 65, no. 7, pp. 5324-5334, Jul. 2018.

\section{BIOGRAPHIES}

Lynn Verkroost was born in Antwerp, Belgium, in 1994. She received the M.Sc. degree in electromechanical engineering from Ghent University, Ghent, Belgium, in 2017. Since then, she has been with the Electrical Energy Laboratory, Department of Electrical Energy, Metals, Mechanical Constructions and Systems, Ghent University, where she is currently working toward the $\mathrm{Ph} . \mathrm{D}$. degree in electromechanical engineering. Her current research interests include digital control of converter-fed electrical machines, and fault-tolerant control.

Joachim Druant was born in Ieper, Belgium, in 1990. He received the M.Sc. degree in electromechanical engineering from Ghent University, Ghent, Belgium, in 2013. Since then, he has been with the Electrical Energy Laboratory, Department of Electrical Energy, Metals, Mechanical Constructions and Systems, Ghent University, where he is currently working toward the Ph.D. degree in electromechanical engineering. In 2014, he received a Ph.D. Fellowship from the Research Foundation-Flanders (FWO). His current research interests include digital control of converter-fed electrical machines, fault-tolerant control, and modeling and control of electrical variable transmissions.

Hendrik Vansompel was born in Belgium in 1986. He received the masters and $\mathrm{Ph} . \mathrm{D}$. degrees in electromechanical engineering from Ghent University, Ghent, Belgium, in 2009 and 2013, respectively. He is currently a Postdoctoral Research Assistant with the Department of Electrical Energy, Metals, Mechanical Constructions and Systems, Ghent University. His research interests include the design and control of electrical drives.

Frederik De Belie was born in Belgium in 1979. He received the masters degree in electromechanical engineering, in 2002, and the Ph.D. degree in electromechanical engineering, in 2010, both from Ghent University, Ghent, Belgium. He is currently an Associate Professor with the Department of Electrical Energy, Metals, Mechanical Constructions and Systems, Ghent University. His current research interests include modeling theory and control-system theory applied to electrical drives and, in particular, selfsensing control of synchronous machines.

Peter Sergeant received the M.Sc. degree in electromechanical engineering and the Ph.D. degree in engineering sciences from Ghent University, Ghent, Belgium, in 2001 and 2006, respectively. In 2001, he became a Researcher with the Electrical Energy Laboratory, Ghent University. He became a Postdoctoral Researcher at Ghent University in 2006 (Postdoctoral Fellow of the Research Foundation-Flanders) and at Ghent University College in 2008. Since 2012, he has been Associate Professor with Ghent University. His current research interests include numerical methods in combination with optimization techniques to design nonlinear electromagnetic systems, in particular, electrical machines for sustainable energy applications. 